\title{
Corilagin induces apoptosis and autophagy in NRF2-addicted U251 glioma cell line
}

\author{
JILAN LIU $^{1 *}$, XIANYUN QIN $^{1 *}$, WENYUAN MA $^{2}$, SHU JIA $^{1}$, XIAOBEI ZHANG ${ }^{1}$, \\ XINLIN YANG ${ }^{3}$, DONGFENG PAN ${ }^{4}$ and FENG JIN ${ }^{4,5}$
}

\author{
Departments of ${ }^{1}$ Central Laboratory and ${ }^{2}$ Neurosurgery, Affiliated Hospital of Jining Medical University, \\ Jining, Shandong 272029, P.R. China; Departments of ${ }^{3}$ Orthopaedic Surgery, Orthopaedic Research Labs and \\ ${ }^{4}$ Radiology and Medical Imaging, University of Virginia, Charlottesville, VA 22908, USA; \\ ${ }^{5}$ Department of Neurosurgery, Affiliated Hospital of Jining Medical University and \\ Shandong Provincial Key Laboratory of Stem Cells and Neuro-Oncology, Jining, Shandong 272029, P.R. China
}

Received May 26, 2020; Accepted November 25, 2020

DOI: $10.3892 / \mathrm{mmr} .2021 .11959$

\begin{abstract}
Corilagin, extracted from the Euphorbiaceae and Phyllanthus plants, inhibits the growth of a number of types of tumors. Compared with temozolomide, the traditional chemotherapy drug, corilagin has demonstrated stronger antitumor activity. However, the pharmaceutical mechanism of corilagin in glioma remains unclear. Nuclear factor erythroid 2 like 2 (NFE2L2 or NRF2) is positively associated with several types of tumor including glioma. In the present study, NRF2 expression was higher in glioma tissues compared with non-glioma specimens. Therefore, it was hypothesized that corilagin targets NRF2 regulation of U251 cell apoptosis. The present study used Hoechst 33258 staining to demonstrate that corilagin induced glioma cell apoptosis and observed that the expression of the apoptosis-related gene Bcl-2 was reduced. In addition, corilagin induced autophagy and promoted the conversion of light chain 3 (LC3) protein from LC3I to LC3II. NRF2 expression was downregulated by corilagin stimulation. Furthermore, the gene expression pattern following knockdown of NRF2 in U251 cells using siRNA was consistent with corilagin stimulation. Therefore, it was preliminarily concluded that corilagin induces apoptosis and autophagy by reducing NRF2 expression.
\end{abstract}

\section{Introduction}

Gliomas are the commonest type of primary brain tumors with high morbidity, mortality and recurrence in addition to being

Correspondence to: Dr Feng Jin, Department of Neurosurgery, Affiliated Hospital of Jining Medical University and Shandong Provincial Key Laboratory of Stem Cells and Neuro-Oncology, 89 Guhuai Road, Jining, Shandong 272029, P.R. China

E-mail: jinfengsdjn@163.com

*Contributed equally

Key words: corilagin, nuclear factor erythroid 2 like 2, glioma, autophagy, apoptosis one of the challenging disorders to treat among all central nervous system diseases (1). According to histopathological features, the World Health Organization classifies gliomas into four grades. The latest classification stated that low-grade gliomas (LGG) can be completely cured if effective treatment is administered (2). Glioblastoma multiforme (GBM), the most malignant glioma, is difficult to be cured using surgery or chemotherapy $(2,3)$. Patients with glioma also face the problem of chemotherapy drug resistance clinically. Due to high drug resistance, several chemotherapeutic drugs traditionally used for treating glioma, including temozolomide, are often not effective (4). Therefore, development of more efficient drugs with novel mechanisms of action is required.

Schmidt and Lademann (5) first isolated corilagin in 1951; Kakiuchi et al (6) discovered in 1985 that corilagin exhibits reverse transcriptase activity against RNA tumor viruses. Over the past decades, a number of studies have reported various pharmacological activities of corilagin, including anti-tumor activity (7), anti-oxidation activity (8), liver and lung protection $(9,10)$ and anti-inflammatory activity (11). Other studies have noted that corilagin can inhibit various tumors such as gastric (12), liver (13), breast (14) and ovarian cancers (15). Corilagin not only directly inhibits tumor cell proliferation, but also indirectly exerts anti-tumor effects by enhancing the effect of traditional chemotherapy drugs (16). A recent study reported that corilagin can induce tumor cell apoptosis through mitochondrial damage (14). According to the potential application value of corilagin for the treatment of glioma, the present study identified that corilagin could induce apoptosis of glioma cells.

Autophagy, also known as type II cell death, is regulated by autophagy-related genes (17). It is the cellular process in which damaged organelles and macromolecules are transported to lysosomes for degradation to maintain the stability of the intracellular environment (18). Autophagy usually occurs at a low level but increases rapidly when adenosine triphosphate energy is depleted, reactive oxygen species (ROS) are released and mitochondrial membrane channels are opened (19). Microtubule-associated protein light chain 3 (LC3) is recognized as a universal marker for autophagosome detection. It 
has two molecular forms, LC3I and LC3II. LC3II is a crucial molecule in the formation of autophagosomes and is widely used to detect the activation of autophagy. SQSTM1/p62 is a connexin that guides the degradation of ubiquitinated proteins into autophagosomes, which can be degraded along with autophagic flow; p62 accumulates when autophagic flow is disordered (20). The present study observed that the rate of conversion of LC3I into LC3II increases and the amount of p62 protein decreases following corilagin activation in U251 glioma cells, suggesting that corilagin induces autophagy.

Nuclear factor erythroid 2 like 2 (NFE2L2 or NRF2) is a protective factor against cellular stress response (21). Studies (21-23) have demonstrated that NRF2 activates various gene transcriptional factors to protect cells against oxidative stress and endogenous and exogenous stimulation; therefore, NRF2 is considered a protective transcription factor for cells (24). Evidence has indicated that NRF2 inhibits cell apoptosis $(25,26)$. In addition, NRF2 is demonstrated to be associated with metastasis and resistance mechanisms of cholangiocarcinoma (27). As a result of these advantages, cancer cells with sustained NRF2 activation often develop 'NRF2 addiction' and display a malignant phenotype, leading to a poor prognosis among patients with cancer (28). Previous studies have demonstrated that U251 cells exhibit aberrant activation of NRF2 and knockdown of NRF2 inhibits the proliferation and growth of U251 cells in a mouse xenograft model $(28,29)$; therefore the present study termed U251 cells NRF2-addicted. Inhibition of NRF2 is a promising therapeutic strategy for NRF2-addicted cancers and the development of NRF2 inhibitors is urgent (28). Under normal conditions, Kelch-like epichlorohydrin-related protein 1 (Keap1) binds to NRF2 protein and the complex is degraded rapidly by the $26 \mathrm{~S}$ proteasome, with the NRF2 protein escaping capture by the Keap1 accumulated in the nucleus (30). This process can be regulated by the autophagy activity in cells. As a substrate for autophagy, SQSTM1/p62 binds to Keap1 and causes NRF2 and Keap1 to separate (30). Subsequently, NRF2 enters the nucleus, activates the transcription of various antioxidant genes and inhibits the expression of oxidative-stress-related proteins (30). The present study preliminarily confirmed that NRF2 is highly expressed in glioma cancer. In addition, corilagin stimulated the U251 cell line, which increased autophagy activity, enhanced p62 degradation and reduced NRF2 protein expression; The present study concluded that the promotion of autophagy by corilagin induced the downregulation of NRF2 expression.

\section{Materials and methods}

Reagents and antibodies. Corilagin (98\% purity) was purchased from Shanghai Yuanye Bio-Technology Co., Ltd., dissolved with phosphate buffer saline (Beijing Solarbio Science \& Technology Co., Ltd.) to prepare stock solution $(4 \mathrm{mg} / \mathrm{ml})$ and stored at $4^{\circ} \mathrm{C}$. Dulbecco's modified Eagle's medium high glucose (DMEM) medium, RPMI-1640 Medium and Earle's Balanced Salt Solution (EBSS) were obtained from HyClone (Cytiva). Fetal bovine serum (FBS) were purchased from HyClone (Cytiva), penicillin and streptomycin were purchased from Beijing Solarbio Science \& Technology Co., Ltd. The trypsin solution was obtained from Gibco (Thermo
Fisher Scientific, Inc.). RIPA Lysis Buffer, phenylmethylsulfonyl fluoride, BCA protein assay kit and 5X loading buffer were purchased from Beyotime Institute of Biotechnology. $\beta$-actin (cat. no. AC026, monoclonal, rabbit anti-human) antibody was obtained from ABclonal Biotech Co., Ltd. NRF2 (cat. no. SC-365949, monoclonal, mouse anti-human) antibody was obtained from Santa Cruz Biotechnology, Inc. Bcl-2 (cat. no. 4223, monoclonal, rabbit anti-human) and LC3 (cat. no. 12741, monoclonal, rabbit anti-human) antibody were purchased from Cell Signaling Technology, Inc. P62 (cat. no. BA2849, polyclonal, rabbit anti-human) antibody was obtained from Wuhan Boster Biological Technology, Ltd. ECL chemiluminescence solution and $0.2 \mu \mathrm{m}$ thickness PVDF membrane were purchased from EMD Millipore. The primers of NRF2 and Bcl-2 were obtained from Beijing Liuhe BGI. Horseradish peroxidase-labeled secondary antibody (anti-rabbit IgG: cat. no. AS014; anti-mouse IgG: AS003) were purchased from ABclonal Biotech Co., Ltd. Coralite488-labeled secondary antibody (cat. no. SA00013-2) was purchased from ProteinTech Group, Inc.

Collection of patient samples. The present study was approved by the Ethics Committee of Affiliated Hospital of Jining Medical University (Jining, China; approval no. 2019C008). Each participant (or their legal representative) provided written informed consent. The 15 glioma tissues were obtained from patients with glioma following surgical resection at Affiliated Hospital of Jining Medical University. The inclusion criteria were patients diagnosed with glioma and treated with surgical resection. In addition, none of the patients received chemotherapy prior to surgery. The exclusion criteria were patients with unconfirmed pathology and/or patients with incomplete data records. The nine non-glioma specimens were obtained from patients with cerebral hemorrhage $(\mathrm{CH})$ and Traumatic Brain Injury (TBI) following surgical resection at Affiliated Hospital of Jining Medical University. All tissues were promptly snap-frozen in liquid nitrogen following surgical resection and stored at $-80^{\circ} \mathrm{C}$ refrigerator until further use.

Cell culture. The U251 cell line was obtained from the Shandong Provincial Key Laboratory of Stem Cells and Neuro-oncology. U251 cells were cultured in DMEM supplemented with $10 \% \mathrm{FBS}, 100 \mathrm{U} / \mathrm{ml}$ penicillin and $100 \mu \mathrm{g} / \mathrm{ml}$ streptomycin. The U251 cells were cultured in a humidified atmosphere at $37^{\circ} \mathrm{C}$ under $5 \% \mathrm{CO}_{2}$. According to the Cellosaurus reference website (https://web.expasy.org/cellosaurus/CVCL_2219), the U251 cell line used was U251 MG and was authenticated using short tandem repeat analysis. U251 cells were seeded at $1 \times 10^{5}$ per well into a 6 -well plate and stimulated by corilagin at different concentrations of $0,25,50,100 \mu \mathrm{g} / \mathrm{ml}$ in a $37^{\circ} \mathrm{C}$ incubator for $48 \mathrm{~h}$ beginning at the second day. EBSS was added to control group cells to make positive control group in $37^{\circ} \mathrm{C}$ incubator for $2 \mathrm{~h}$. Cells were collected for reverse transcription-quantitative (RT-q) PCR, western blot analysis, immunofluorescence and Hoechst 33258 staining.

RNA extraction and RT-qPCR. Cells were harvested for RNA extraction when at $80-90 \%$ confluence. The total RNA was isolated from U251 cells with TRIzol ${ }^{\circledR}$ reagent (Thermo Fisher Scientific, Inc.) following the manufacturer's protocol. Total RNA 
$(1.5 \mu \mathrm{g})$ was prepared for reverse transcription using a FastQuant RT kit (Tiangen Biotech Co., Ltd.) to obtain the cDNA. The cDNA and SYBR-Green mixture (CoWin Biosciences) were used following the manufacturer's protocol for RT-qPCR. The machine used was from Bio-Rad Laboratories, Inc. and the RT-qPCR program was as follows: $95^{\circ} \mathrm{C} 10 \mathrm{~min}, 95^{\circ} \mathrm{C} 10 \mathrm{sec}$ and $60^{\circ} \mathrm{C}$ $30 \mathrm{sec}\left(45\right.$ cycles), $95^{\circ} \mathrm{C} 1 \mathrm{~min}, 55^{\circ} \mathrm{C} 1 \mathrm{~min}, 55^{\circ} \mathrm{C} 10 \mathrm{sec}, 95^{\circ} \mathrm{C}$ $5 \mathrm{sec}$. The $2^{-\Delta \Delta \mathrm{Cq}}$ method was used for data analysis (31) and the relative expression was normalized to $\beta$-actin. The primers sequences used for the experiment were: $\beta$-actin, forward, 5'-GAA GTGTGACGTGGACATCC-3' and reverse, 5'-CCGATCCAC ACGGAGTACTT-3'; NRF2, forward, 5'-GAGAGCCCAGTCTT CATTGC-3' and reverse, 5'-TGCTCAATGTCCTGTTGCAT-3'; Bcl-2, forward, 5'-GGTGGGGTCATGTGTGTGG-3' and reverse, 5'-CGGTTCAGGTACTCAGTCA TCC-3'. At least three independent assays were performed in triplicate.

Western blot analysis. Following corilagin stimulation, U251 cells were harvested for lysis using RIPA lysate buffer. Lysates were incubated on ice for $>30 \mathrm{~min}$ and centrifuged at $12,000 \mathrm{x} \mathrm{g}$ for $15 \mathrm{~min}$ at $4^{\circ} \mathrm{C}$. The supernatant was transferred to a new Eppendorf tube and the precipitate discarded. Protein concentration was detected by a BCA determination kit. Proteins $(50 \mu \mathrm{g})$ were separated by $12 \%$ SDS-PAGE and transferred onto a PVDF membrane. Membranes were blocked with $5 \%$ skimmed milk for $1 \mathrm{~h}$ at room temperature and incubated with primary antibodies at a dilution of 1:1,000 overnight at $4^{\circ} \mathrm{C}$. The membranes were incubated with a horseradish peroxidase (HRP)-conjugated secondary (1:5,000 dilution) at $37^{\circ} \mathrm{C}$ for $1 \mathrm{~h}$. Blots were detected using ECL chemiluminescent reagents. The relative protein expression of genes was quantified by ImageJ v.1.46r software (National Institutes of Health) using $\beta$-actin as control, Data are shown as mean \pm standard deviation from at least three independent experiments. $\mathrm{P}<0.05$ was considered to indicate a statistically significant difference.

Hoechst 33258 staining. Following corilagin stimulation, U251 cells were washed with PBS twice at room temperature. The cells were fixed with methanol for $10 \mathrm{~min}$ and incubated with Hoechst 33258 staining reagent for $10 \mathrm{~min}$ at room temperature. A confocal microscope (Zeiss LSM800; Zeiss AG) was used to detect the apoptosis levels at x100 magnification and the apoptotic cells appeared as dense bright blue. The experiments were carried out three times, individually.

Immunofluorescence staining. Following corilagin stimulation, U251 cells were washed with PBS three times and fixed with $4 \%$ paraformaldehyde for $10 \mathrm{~min}$ at room temperature. Cell membrane was penetrated using PBS containing $0.1 \%$ Triton X-100 and blocked with $10 \%$ FBS at room temperature. Cells were incubated with LC3B primary antibody (1:200 dilution) at room temperature for $2 \mathrm{~h}$. The cells were washed three times and incubated with coralite488-labeled secondary antibody (1:100) at room temperature for $30 \mathrm{~min}$. Following washing with PBS for three times, the cells were incubated with DAPI for $10 \mathrm{~min}$ at room temperature. Finally, the slides were sealed with anti-fluorescent quencher to delay fluorescence quenching. All experiments were performed in triplicate and repeated at least three times.
Cell transfection. U251 cells were seeded in 6-well plates (2x105/well) until reached $30-40 \%$, before transfection, the transfection reagent Lipofectamine ${ }^{\circledR} 2000$ (Invitrogen; Thermo Fisher Scientific, Inc.), $10 \mathrm{nM}$ negative control (NC) or $10 \mathrm{nM}$ small interfering (si)RNAs were mixed and incubated for $20 \mathrm{~min}$, and then added to the wells with serum-free medium. The NC and siRNAs were synthesized by Shanghai GenePharma Co.,Ltd.Sequence of NC was: 5'-UUCUCCGAAC GUGUCACGUTT-3'; sequence of siRNAs were: siNrf2-1, 5'-GGUUGAGACUACCAUGGUUTT-3'; siNrf2-2, 5'-GAC AGAAGUUGACAAUUAUTT-3'; siNrf2-3, 5'-CCAGAACAC UCAGUGGAAUTT-3'. Following transfection for $72 \mathrm{~h}$, cells were harvested for further study.

GEPIA dataset. GEPIA is an interactive web server for analyzing the RNA sequencing expression data that includes 9,736 tumors and 8,587 normal samples from The Cancer Genome Atlas and the Genotype-Tissue Expression projects, using the output of a standard processing pipeline for RNA sequencing data. GEPIA provides customizable functions such as tumor/normal differential expression analysis, profiling according to cancer types or pathological stages, patient survival analysis, similar gene detection, correlation analysis, and dimensionality reduction analysis. GEPIA dynamically plots expression profiles of a given gene according to user-defined sample selections and methods. The results can be presented in box plots. In addition, GEPIA plots gene expression by pathological stages based on the TCGA clinical annotation. GEPIA performs survival analysis based on gene expression levels. This function allows users to select their custom cancer types for survival analysis. For example, to examine the survival curves of an input gene in glioma, we select both GBM and LGG for the survival analyses. GEPIA uses log-rank test for the hypothesis evaluation. The hazard ratio and the $95 \%$ confidence interval information can also be included in the survival plot (32).

Statistical analysis. All data were expressed as mean \pm standard deviation and analyzed by one-way ANOVA followed by Bonferroni's post hoc test using GraphPad Prism v.5 (GraphPad Software, Inc.). The error bars were derived from at least three independent experiments. $\mathrm{P}<0.05$ was considered to indicate a statistically significant difference.

\section{Results}

NRF2 is overexpressed in glioma tissues and is negatively correlated with patient survival. The nine non-glioma specimens were taken from patients with $\mathrm{CH}$ or $\mathrm{TBI}$ requiring intracranial decompression and the 15 tumor tissues were taken from patients with glioma. The basic characteristics of these 24 brain tissues are listed in Tables I and II. Total proteins were extracted the from these tissues and it was discovered by using western blotting that NRF2 was overexpressed in glioma tissues compared with non-glioma specimens (Fig. 1A and B). Information from a large sample database (Gene Expression Profiling Interactive Analysis, GEPIA) revealed that NRF2 was overexpressed in GBM and LGG (Fig. 1C). NRF2 was negatively correlated with patient survival using GEPIA. The low NRF2 expression group demonstrated higher percent survival rates compared with the high NRF2 expression group (Fig. 1D). 
Table I. Characteristics of non-glioma patient samples.

\begin{tabular}{lccccccccc}
\hline Number & C1 & C2 & C3 & C4 & C5 & C6 & C7 & C8 & C9 \\
\hline Sex & M & F & M & M & F & M & M & F & F \\
Age (years) & 40 & 43 & 69 & 44 & 27 & 51 & 31 & 44 & 55 \\
Source & CH & TBI & CH & CH & CH & CH & TBI & CH & CH \\
\hline
\end{tabular}

M, male; F, female; CH, Cerebral hemorrhage; TBI, Traumatic Brain Injury

Table II. Clinicopathological characteristics of glioma patient samples.

\begin{tabular}{lccccccccccccccc}
\hline Number & T1 & T2 & T3 & T4 & T5 & T6 & T7 & T8 & T9 & T10 & T11 & T12 & T13 & T14 & T15 \\
\hline Sex & F & F & M & F & M & F & F & M & F & F & M & F & M & M & M \\
Age (years) & 67 & 72 & 67 & 40 & 60 & 50 & 67 & 40 & 60 & 68 & 44 & 53 & 39 & 68 & 70 \\
WHO Grade & IV & IV & III & IV & IV & IV & IV & III & III-IV & III & III & IV & IV & IV & IV \\
\hline
\end{tabular}

Corilagin downregulates the expression of NRF2 in the U251 glioma cell line. The present study demonstrated that corilagin, an anti-glioma drug, possessed a clear ability to inhibit the proliferation of glioma cells (33). To determine whether it inhibits NRF2 express ion to suppress glioma cells, the regulation effect exerted by corilagin on NRF2 was investigated. Following the use of corilagin to stimulate U251 cells, the protein expression of NRF2 was detected though western blotting. The protein expression of NRF2 was weakened in the corilagin treatment group compared with the control group (Fig. 2A and B). Following stimulation of U251 cells by using $100 \mu \mathrm{g} / \mathrm{ml}$ corilagin, the protein expression of NRF2 was detected though immunofluorescence. The fluorescence intensity of NRF2 was weakened in the corilagin treatment group (Fig. 2C).

Corilagin induces apoptosis of the U251 glioma cell line. To determine whether corilagin exerts other effects on U251 glioma cells, the following experiments were performed. Different concentrations of corilagin $(0,25,50$ and $100 \mu \mathrm{g} / \mathrm{ml})$ were used to stimulate cells for $48 \mathrm{~h}$ and the level of cell apoptosis was detected using Hoechst 33258 staining. The results demonstrated that cells appeared bright blue with the intensity of the color increasing as concentration increased (Fig. 3A). This indicated that corilagin induced apoptosis of the U251 glioma cells. To further explore the induced-apoptosis, the results were verified at the molecular level. The corilagin-stimulated cells were harvested and the protein expression of $\mathrm{Bcl}-2$ was detected using western blotting. The result indicated that the protein expression of $\mathrm{Bcl}-2$ was reduced by the increased concentration of corilagin (Fig. 3B). The mRNA expression of $\mathrm{Bcl}-2$ was detected using RT-qPCR and the results indicated that the mRNA expression of Bcl-2 was also reduced by the increasing concentration of corilagin (Fig. 3C).

Corilagin induces the autophagy of the U251 glioma cell line. Whether corilagin regulated cell autophagy was investigated. EBSS is a positive inducer of autophagy. Following stimulation of U251 cells using EBSS and $100 \mu \mathrm{g} / \mathrm{ml}$ corilagin, the cell autophagy-related protein LC3II was detected though immunofluorescence. The level of LC3II increased significantly in the EBSS and corilagin treatment groups (Fig. 4A) by immunofluorescence, whereas the expression of P62 decreased compared with the control group, as observed using western blotting (Fig. 4B). Although the decrease of P62 appears slight, no less than three experiments prove that the decrease is statistically significant. Therefore, the level of autophagy in the corilagin treatment group was higher compared with the control group. The relative protein expression levels of P62 and the ratio of LC3II to LC3I are illustrated in Fig. 4C. In addition, the mRNA level of P62 was reduced in the corilagin-treated group (Fig. 4D) confirming that the level of autophagy in the Corilagin treatment group was also greater than that in the control group.

NRF 2 regulates apoptosis and autophagy. To demonstrate whether corilagin induced apoptosis and autophagy via inhibiting NRF2, small interfering (si)RNAs were used to knock down the NRF2 expression in U251 cell line. In the present study, three siRNAs sequences of NRF2 were designed, but only one of these siRNA sequences significantly inhibited the expression of NRF2. It was discovered that as the expression of NRF2 was suppressed, the expression of P62 and Bcl-2 were also suppressed and the ratio of LC3IIto LC3Iis increased (Fig. 5A and B). This result is consistent with the effect of corilagin on stimulating cells. As mentioned above, corilagin clearly inhibited NRF2 expression, so it was hypothesized that the inhibitory effect of corilagin on cells was at least partly dependent on NRF2 inhibition.

\section{Discussion}

The present study demonstrated that corilagin induced apoptosis and autophagy of the NRF2-addicted U251 glioma cell line and reduced NRF2 expression. The significance of the 
A
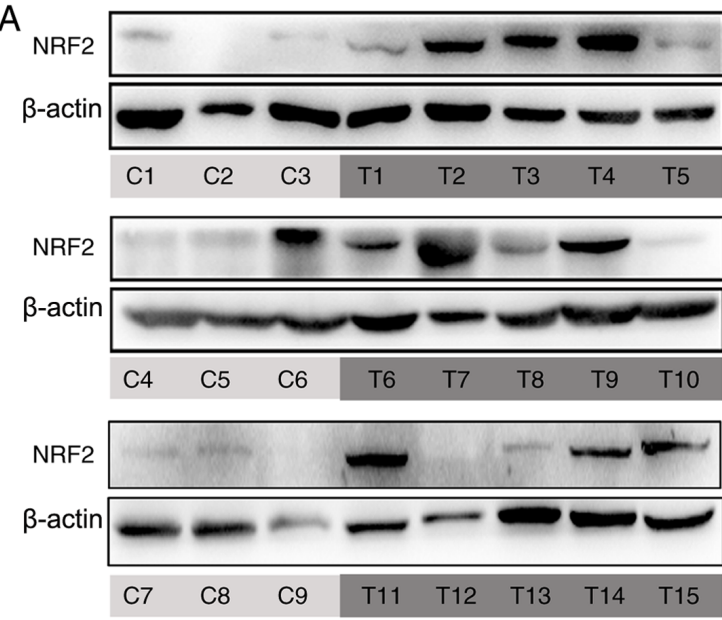

C

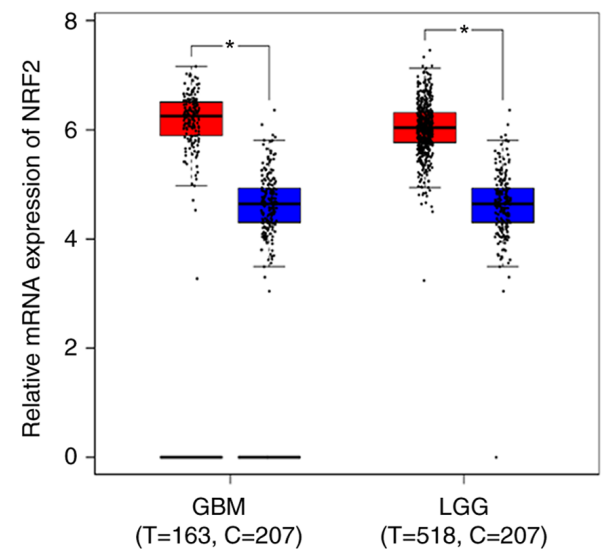

B
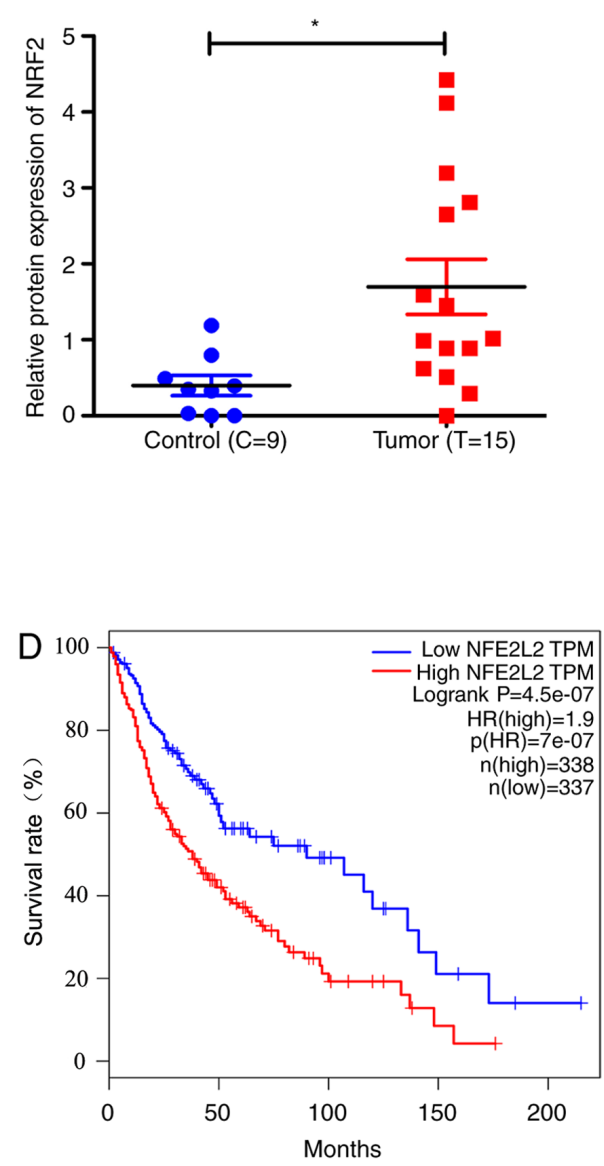

Figure 1. NRF2 is overexpressed in glioma tissues and negatively correlates with the survival rates of patients. (A) NRF2 was overexpressed in the 15 glioma tissues compared with the 9 control samples by western blotting. (B) The relative protein expression level of NRF2 was calculated using $\beta$-actin as the loading control ( $\mathrm{P}<0.05)$. (C) The NRF2 mRNA expression profiles in control brain tissue ( $\mathrm{n}=207)$, GBM (T=163) and LGG (T=518) from a data set from GEPIA; "P<0.05. (D) The difference between the low and high NRF2 expression groups. There were 337 patients with low expression of NRF2 and there were 338 patients with high expression of NRF2 (GEPIA). HR demonstrated that the risk of mortality in the NRF2 high expression group was 1.9 times higher compared with that of the NRF2 low expression group. NRF2, nuclear factor erythroid 2 like 2; T, glioma tissues; C, control samples; GBM, glioblastoma multiforme; LGG, low grade glioma; GEPIA, Gene Expression Profiling Interactive Analysis; HR, Hazard Ratio.

target gene NRF2 in the anti-glioma role of corilagin was identified. The present study aimed to discover a new function of corilagin as a novel antitumor drug and its key target. It also provided a new theoretical basis for tumor cell chemoresistance.

Overcoming chemotherapy resistance is a challenging problem in glioma treatment. Temozolomide is expensive and has poor clinical efficacy (34); the development of new anticancer drugs is thus an urgent matter. A number of studies have demonstrated numerous pharmacological properties of corilagin in vivo and in vitro. In vivo experiments have revealed that corilagin inhibits growth of tumors, such as hepatocellular carcinoma and ovarian cancer, in an athymic implant tumor model $(35,36)$. Due to the blood brain barrier (BBB), animal experiments are crucial for researching anti-glioma chemotherapy drugs. Corilagin has three phenolic rings for $\pi-\pi$ stacking interactions with some amino acid residues and exhibits excellent amyloid- $\beta(A \beta)$ oligomer selectivity with a high binding affinity $(37,38)$. The primary influence driving Alzheimer's disease (AD) pathogenesis is accumulation of $A \beta$ in the brain, therefore, corilagin demonstrates the potential to alleviate AD (37-39). Although the evidence (37-39) support the view that corilagin can pass through the $\mathrm{BBB}$, animal experiments are required to support the conclusion of the present study. In vitro, corilagin exhibits strong inhibitory activity against viruses and microorganisms $(40,41)$. Staphylococcus aureus, well-known for its drug resistance, is inhibited by corilagin at a minimum inhibitory concentration of $25 \mu \mathrm{g} / \mathrm{ml}(42)$. The anti-microorganism activity of corilagin is expected to solve the problem of bacterial drug resistance (43).

Thus, corilagin regulates several signal pathways to participate in biological activities (44). In vivo and in vitro experiments demonstrate that corilagin prevents herpes simplex virus-1 encephalitis by inhibiting the Toll-like receptor 2 signaling pathways (45). Corilagin reduces the release of TNF- $\alpha$, which is considered to stimulate the growth and progression of early malignant tumor cells $(46,47)$. Corilagin attenuates radiation-induced brain injury in mice by inhibiting the expression of NF- $\kappa \mathrm{B}$ and TNF- $\alpha$ (48). Our team demonstrated that corilagin inhibits the proliferation of glioma cells by blocking the NF- $\mathrm{KB}$ pathway (33). However, the present study demonstrated the inhibitory effect of NRF2-mediated corilagin on glioma cells, enriching the anti-tumor mechanism of corilagin, from the perspective of apoptosis and autophagy. 


\section{A}

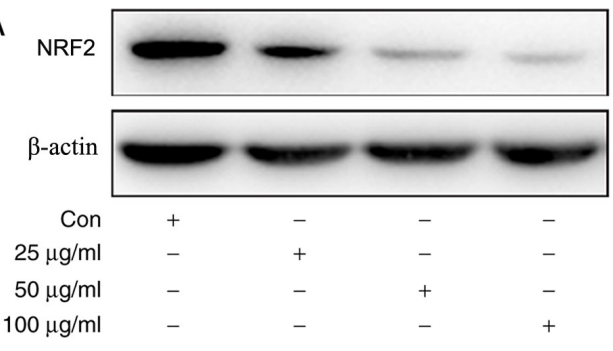

$\mathrm{B}$

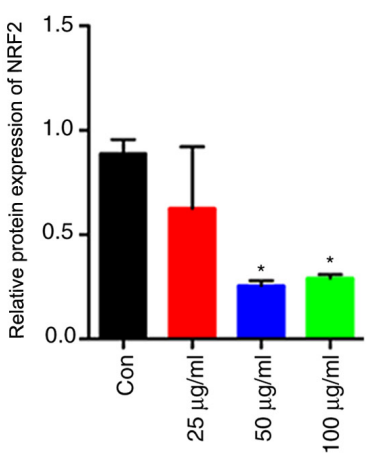

C
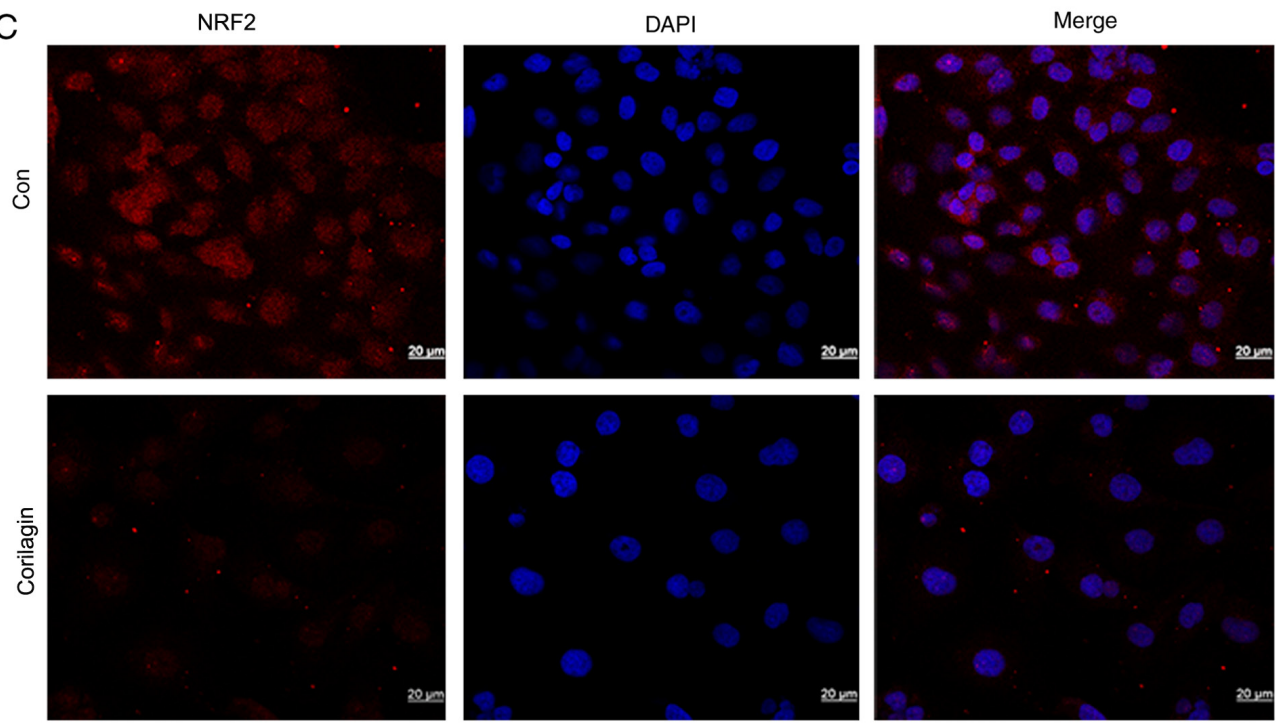

Figure 2. Corilagin downregulates the expression of NRF2. (A) Following stimulation of U251 cells by $100 \mu \mathrm{g} / \mathrm{ml} \mathrm{corilagin}$ for $48 \mathrm{~h}$, the protein expression of NRF2 was detected by western blotting and (B) the relative protein expression level of NRF2 was calculated using $\beta$-actin as the loading control $\left({ }^{*} \mathrm{P}<0.05\right)$. (C) Following stimulation of U251 cells by $100 \mu \mathrm{g} / \mathrm{ml}$ corilagin for $48 \mathrm{~h}$, the protein expression of NRF2 was detected by immunofluorescence. Scale bar, $20 \mu \mathrm{m}$. NRF2, nuclear factor erythroid 2 like 2; con, control.

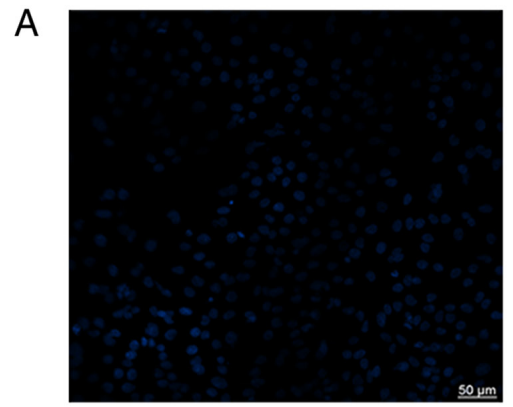

Con

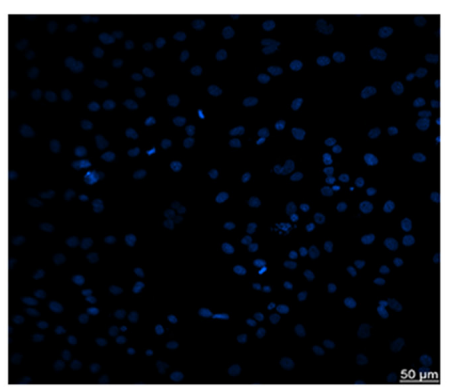

$50 \mu \mathrm{g} / \mathrm{ml}$

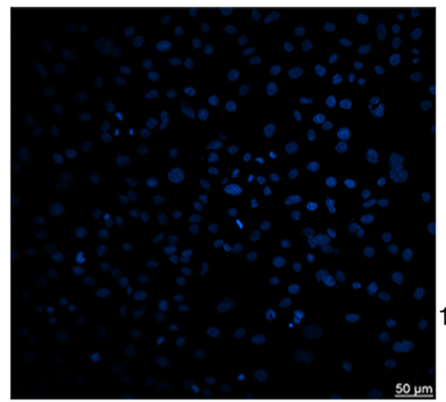

$25 \mu \mathrm{g} / \mathrm{ml}$

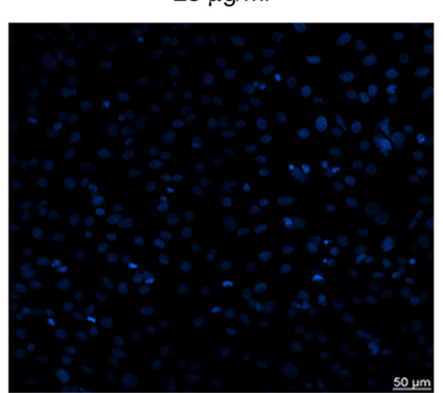

$100 \mu \mathrm{g} / \mathrm{ml}$

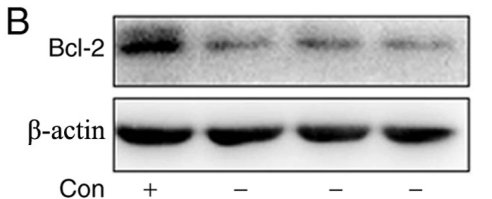

$25 \mu \mathrm{g} / \mathrm{ml} \quad-\quad+\quad+\quad-\quad-$

$50 \mu \mathrm{g} / \mathrm{ml} \quad-\quad-\quad+\quad+$

$100 \mu \mathrm{g} / \mathrm{ml} \quad-\quad \ldots$

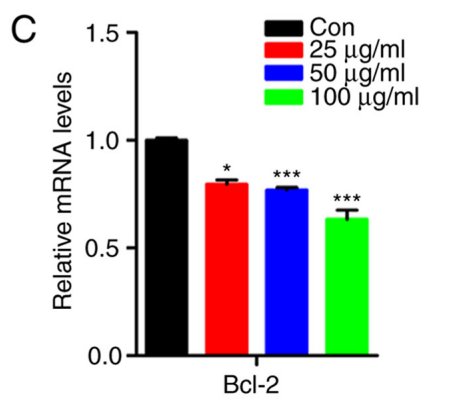

Figure 3. Corilagin induces the apoptosis of U251 glioma cell line. (A) Hoechst 33258 staining showed that the level of cell apoptosis following adding different concentrations of corilagin $(0,25,50$ and $100 \mu \mathrm{g} / \mathrm{ml})$ for $48 \mathrm{~h}$ to U251 cells. Scale bar, $50 \mu \mathrm{m}$. (B) Western blotting identified the protein expression level of Bcl-2 following adding different concentrations of corilagin $(0,25,50$ and $100 \mu \mathrm{g} / \mathrm{ml})$ for $48 \mathrm{~h}$ to U251 cells. (C) The mRNA expression of Bcl-2 was detected in corilagin-stimulated cells by reverse transcription-quantitative PCR normalized to $\beta$-actin $\left({ }^{*} \mathrm{P}<0.05 ;{ }^{* * *} \mathrm{P}<0.001\right)$. con, control. 

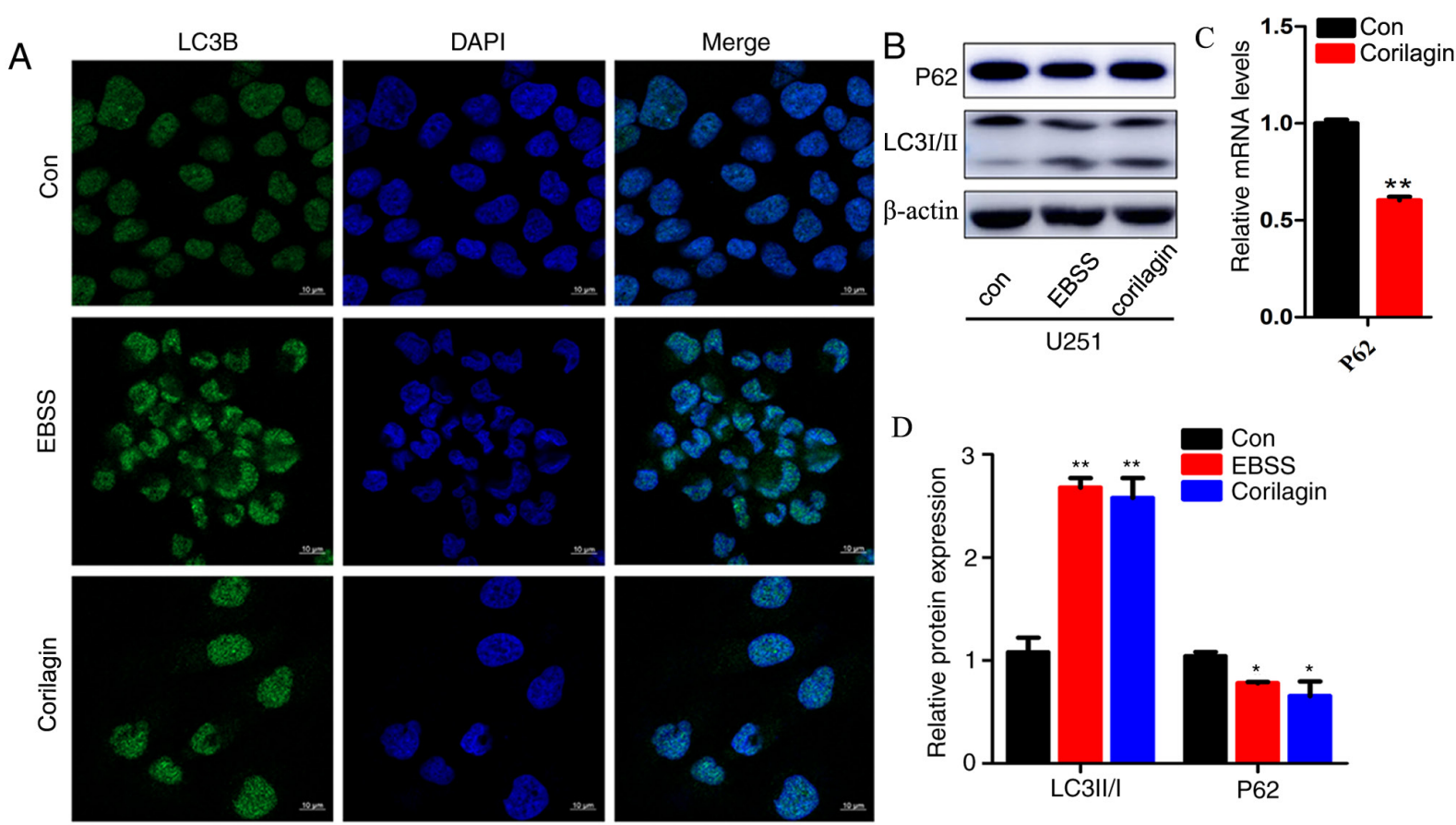

$\mathrm{D}$

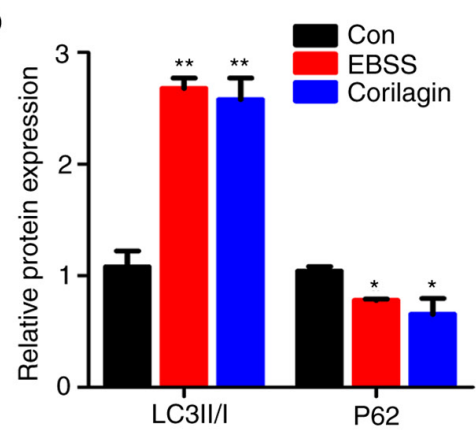

Figure 4. Corilagin induces the autophagy of U251 glioma cell line. (A) Following stimulation of U251 cells by EBSS and $100 \mu \mathrm{g} / \mathrm{ml}$ corilagin, cell autophagy-related protein LC3B were detected by immunofluorescence. Scale bar, $10 \mu \mathrm{m}$. (B) Western blotting demonstrated that the protein level of LC3II in the EBSS and corilagin treatment group were increased and the expression of P62 was significantly decreased. (C) The mRNA expression level of P62 was significantly reduced in the corilagin-treated $(100 \mu \mathrm{g} / \mathrm{ml})$ group as determined by reverse transcription-quantitative PCR normalized to $\beta$-actin $\left({ }^{* *} \mathrm{P}<0.01\right)$. (D) The relative protein ratio of LC3II/LC3I and P62 were calculated using $\beta$-actin as the loading control $\left({ }^{* *} \mathrm{P}<0.01\right.$; $\left.{ }^{*} \mathrm{P}<0.05\right)$. EBSS, Earle's Balanced Salt Solution; LC3, light chain 3; con, control;
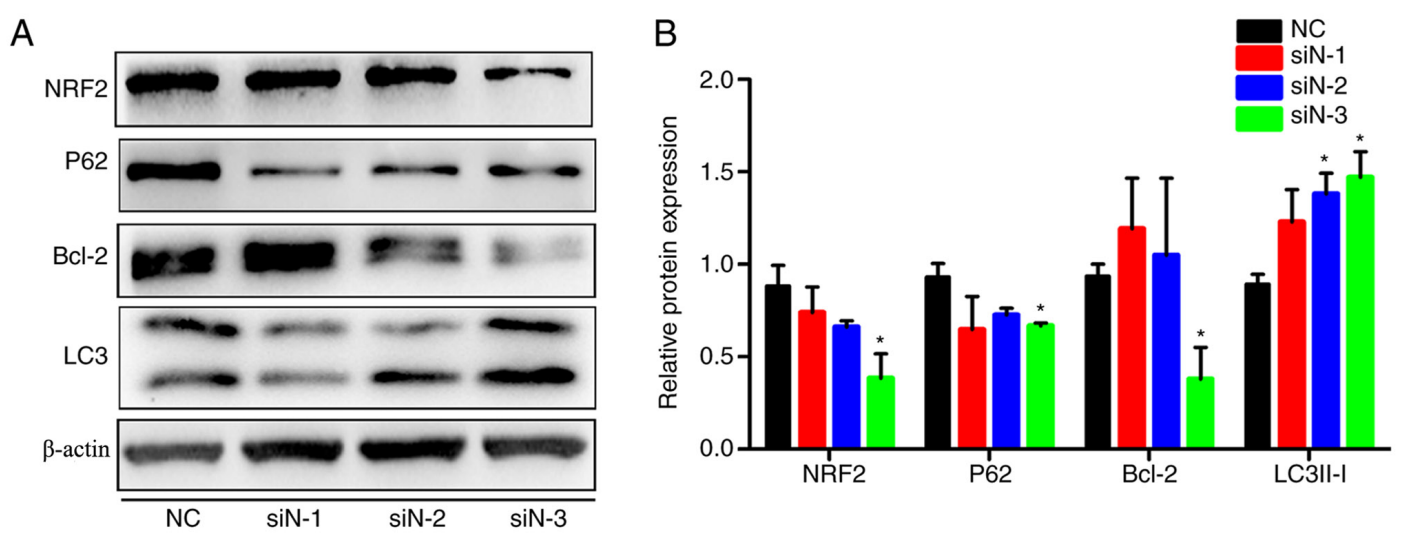

Figure 5. NRF2 regulates apoptosis and autophagy. (A) The U251 cells were transfected with siRNAs by Lipofectamine ${ }^{\circledR} 2000$. The protein expression of NRF2, P62, Bcl-2 and LC3 were detected by western blotting. (B) The relative protein quantitative analysis of the ratio LC3II/LC3I, P62, Bcl-2 and NRF2 were calculated using $\beta$-actin as the loading control ("P<0.05). NRF2, nuclear factor erythroid 2 like 2; con, control; si, short interfering; $\mathrm{NC}$, negative control; siN, siRNA of NRF2.

NRF2 mainly regulates transcriptional activation through antioxidant response elements (AREs) present in the promoters of NRF2 target genes (49). Studies (50-52) have revealed that NRF2 is overexpressed in the KB-derived drug-resistant cancer cell panel. Silencing NRF2 expression can enhance the drug sensitivity of chemoresistant cells and NRF2 activator can enable cells to acquire drug resistance (50). In addition, a previous study demonstrated that disulfiram can selectively inhibit the NRF2 and NF- $\kappa \mathrm{B}$ signaling pathways of leukemia stem cells, restricting their proliferation and inducing apoptosis (53). Our team previously demonstrated that corilagin possesses a strong inhibitory effect on glioma stem cells (33), however, whether glioma stem cells are more addictive to NRF2 than glioma cells requires further research. The present study mainly focused on the regulation of corilagin in glioma cells by inhibiting NRF2 expression. It was demonstrated that NRF2 was highly expressed in glioma. That glioma stem cells are addictive to NRF2 $(28,29)$ supports the conclusion of the present study to some extent. Nevertheless, the small samples size remains a limitation of the present study. In theory, the larger the sample size, the more accurate the research results. When the sample size is not large enough, it is more difficult to use the limited data to prove the significance of the NRF2 overexpression with glioma. Therefore, increasing the samples 
size needs be addressed in future research. With enough samples, the detection of NRF2 expression can be performed to provide more evidence to support the hypothesis of the present study. Previous studies have investigated the mechanism by which corilagin regulates microRNA $(54,55)$. One reported that corilagin reduces the expression of miR-21 and inhibits schistosomiasis-induced hepatic fibrosis (54). Previous studies $(33,42,44-48,54)$ have demonstrated that corilagin regulates numerous pathways and possesses various biological properties, including anti-viral, anti-bacterial and anti-cancer.

Cell apoptosis is considered to be cell death type I, with the ability to maintain cell fate and homeostasis (56). Apoptotic procedures are disrupted during tumorigenesis. The Bcl-2 family of proteins regulate cell apoptosis (57). The family includes proapoptotic proteins, such as Bad, Bax and Bid and antiapoptotic proteins, such as Bcl-2 and Bcl-XL. The balance between proapoptotic and antiapoptotic proteins determines the progress of apoptosis (57). The present study focused on the anti-apoptotic protein Bcl-2 and demonstrated that corilagin induced cell apoptosis through the reduction of Bcl-2 expression. Cell apoptosis is closely associated with autophagy proteins. Autophagy-related (ATG)5 and ATG12, which are necessary for induction of cell autophagy, contribute to the induction of apoptosis. ATG5 interacts with Bcl-XL to promote the release of cytochrome c to regulate cell apoptosis $(58,59)$. The present study focused on NRF2, considered an apoptosis inhibitor. The significance of the NRF2-related apoptosis process is diverse for various physiological processes $(25,60,61)$. For example, a previous study revealed that exposure of non-small-cell lung cancer cells to ionizing radiation induces cell apoptosis and knocking down NRF2 expression boosts cell apoptosis (25). NRF2 acts as a protective factor against normal tissue damage and possesses a protective effect on brain injury by inhibiting mitochondria-related apoptosis (60). Cisplatin resistance is a significant obstacle in the treatment of tumors and it has been demonstrated that NRF2 expression induces cisplatin resistance in ovarian cancer (61). Several studies have reported that NRF2 inhibits apoptosis by activating AREs and that this process is a protective program against cell death (62-64).

The expression level of NRF2 is inextricably linked to autophagy intensity. A previous study clarifies the relationship between NRF2 and autophagy, demonstrating that autophagic flow disorder leads to p62 accumulation and then detachment of NRF2 from Keap1, which in turn regulates the ARE pathway (65). The present study identified that NRF2 expression was reduced by corilagin stimulation. The ubiquitination pathway is a protein degradation pathway independent of lysosomal degradation (66). The $26 \mathrm{~S}$ proteasome is the major protease responsible for protein degradation (67). Numerous factors, including human telomerase reverse transcriptase (68) and cyclic adenosine monophosphate (69), regulate $26 \mathrm{~S}$ proteasome activity. Whether corilagin inhibits NRF2 expression by regulating $26 \mathrm{~S}$ proteasome activity should be further explored. A previous study reported that corilagin induces autophagy in breast cancer cells by inhibiting Akt/mTOR/p70S6K and this induced autophagy can be downregulated by the ROS scavenger $\mathrm{N}$-acetylcysteine (14). This study also showed that the corilagin-induced autophagy of breast cancer cell may depend on ROS generation. Another study demonstrated that corilagin also promotes ROS production and induces autophagy (12). ROS was not detected in the present study, which was one of its limitations. However, a number of studies reveal that NRF2 inhibition induces ROS production (70-72) and these results support the conclusion of the present study that corilagin induced autophagy via downregulating NRF2. In addition, to confirm that the action of corilagin is specific to the reduction of NRF2 expression, it would be more convincing to administer corilagin to NRF2-overexpressing U251 cells to verify the suppression of cell death. The overexpressed vector of NRF2 has not been successfully created yet, and this another limitation to the present study. It is hoped, once the vector is successfully constructed, to remedy this defect.

In conclusion, the present study demonstrated the mechanisms of corilagin in glioma treatment and explored its relationship with NRF2 expression. It was identified that corilagin induced apoptosis and autophagy in the U251 glioma cell line. NRF2 was overexpressed in glioma tissues and its reduced expression contributed to the anti-glioma effect of corilagin. The data from the present study provided solid evidence for corilagin as a candidate drug for glioma treatment.

\section{Acknowledgements}

Not applicable.

\section{Funding}

The present study was supported by the project of Health and Family Planning Commission of Shandong province (grant no. 2017WS513), Supporting Fund for Teachers' research of Jining Medical University (grant nos. JYFC2019FKJ125, JYFC2018FKJ035 and JYFC2018FKJ107), the project of Jining Science and Technology Bureau (grant no. 2016-56-60), Scientific Research Project of Jining Medical University (grant no. JY2015KJ022), Project of Scientific Research Program of Affiliated Hospital of Jining Medical University (grant nos. MP-2018-012, MP-2015-003) and Scientific Research Project of Jining Medical University (grant no. JY2015KJ022).

\section{Availability of data and materials}

All data generated or analyzed during this study are included in this published article.

\section{Authors' contributions}

JL, XQ and FJ conceived and designed the study. JL and XQ conducted the experiments, analyzed the data and drafted the manuscript. WM collected the clinical tissues and participated in protein determination. $\mathrm{SJ}$ and $\mathrm{XZ}$ participated in the data acquisition and analysis. FJ, XY and DP provided experimental guidance and participated in data analysis. All authors read and approved the final manuscript.

\section{Ethics approval and consent to participate}

The present study was approved by the Ethics Committee of Affiliated Hospital of Jining Medical University (Jining, China) and the approval number was 2019C008. The present 
study was performed in accordance with the Declaration of Helsinki and guidelines of the Ethics Committee of Affiliated Hospital of Jining Medical University. Each participant (or their legal representative) provided written informed consent.

\section{Patient consent for publication}

Not applicable.

\section{Competing interests}

The authors declare that they have no competing interests.

\section{References}

1. Chen R,Smith-Cohn M,Cohen AL and Colman H: Glioma subclassifications and their clinical significance. Neurotherapeutics 14 284-297, 2017.

2. Delgado-Lopez PD, Corrales-Garcia EM, Martino J,Lastra-Aras E and Duenas-Polo MT: Diffuse low-grade glioma: A review on the new molecular classification, natural history and current management strategies. Clin Transl Oncol 19: 931-944, 2017.

3. Duffau H and Taillandier L: New concepts in the management of diffuse low-grade glioma: Proposal of a multistage and individualized therapeutic approach. Neuro Oncol 17: 332-342, 2015

4. Chen X, Zhang M, Gan H, Wang H, Lee JH, Fang D, Kitange GJ, He L, Hu Z, Parney IF, et al: A novel enhancer regulates MGMT expression and promotes temozolomide resistance in glioblastoma. Nat Commun 9: 2949, 2018.

5. Schmidt OT and Lademann R: Corilagin, ein weiterer kristallisierter Gerbstoff aus Dividivi. X. Mitteilung über natürliche Gerbstoffe. Justus Liebigs Ann Chem 571: 232-237, 1951.

6. Kakiuchi N, Hattori M, Namba T, Nishizawa M, Yamagishi T and Okuda T: Inhibitory effect of tannins on reverse transcriptase from RNA tumor virus. J Nat Prod 48: 614-621, 1985.

7. Qiu F, Liu L, Lin Y, Yang Z and Qiu F: Corilagin inhibits esophageal squamous cell carcinoma by inducing DNA damage and down-regulation of RNF8. Anticancer Agents Med Chem 19: 1021-1028, 2019

8. Ding Y, Ren D, Xu H, Liu W, Liu T, Li L, Li J, Li Y and Wen A Antioxidant and pro-angiogenic effects of corilagin in rat cerebral ischemia via Nrf2 activation. Oncotarget 8: 114816-114828, 2017

9. Liu FC, Chaudry IH and Yu HP: Hepatoprotective effects of corilagin following hemorrhagic shock are through akt-dependent pathway. Shock 47: 346-345, 2017.

10. Guo S, Fu Y, Xiong S and Lv J: Corilagin protects the acute lung injury by ameliorating the apoptosis pathway. Biomed Pharmacother 95: 1743-1748, 2017.

11. Li HR, Liu J, Zhang SL, Luo T, Wu F, Dong JH, Guo YJ and Zhao L: Corilagin ameliorates the extreme inflammatory status in sepsis through TLR4 signaling pathways. BMC Complement Altern Med 17: 18, 2017.

12. Xu J, Zhang G, Tong Y, Yuan J, Li Y and Song G: Corilagin induces apoptosis, autophagy and ROS generation in gastric cancer cells in vitro. Int J Mol Med 43: 967-979, 2019.

13. Deng Y, Li X, Li X, Zheng Z, Huang W, Chen L, Tong Q and Ming Y: Corilagin induces the apoptosis of hepatocellular carcinoma cells through the mitochondrial apoptotic and death receptor pathways. Oncol Rep 39: 2545-2552, 2018.

14. Tong Y, Zhang G, Li Y, Xu J, Yuan J, Zhang B, Hu T and Song G: Corilagin inhibits breast cancer growth via reactive oxygen species-dependent apoptosis and autophagy. J Cell Mol Med Jun 22: 3795-3807, 2018.

15. Li N, Lin Z, Chen W, Zheng Y, Ming Y, Zheng Z, Huang W, Chen L, Xiao J and Lin H: Corilagin from longan seed: Identification, quantification, and synergistic cytotoxicity on SKOv3ip and hey cells with ginsenoside Rh2 and 5-fluorouracil. Food Chem Toxicol 119: 133-140, 2018

16. Milani R, Brognara E,FabbriE, Finotti A, Borgatti M,Lampronti I, Marzaro G, Chilin A, Lee KK, Kok SH, et al: Corilagin induces high levels of apoptosis in the temozolomide-resistant T98G glioma cell line. Oncol Res 26: 1307-1315, 2018

17. Noguchi M, Hirata N, Tanaka T, Suizu F, Nakajima H and Chiorini JA: Autophagy as a modulator of cell death machinery. Cell Death Dis 11: 517, 2020.
18. Choi AM, Ryter SW and Levine B: Autophagy in human health and disease. N Engl J Med 368: 651-662, 2013

19. Baechler BL, Bloemberg D and Quadrilatero J: Mitophagy regulates mitochondrial network signaling, oxidative stress, and apoptosis during myoblast differentiation. Autophagy 15: 1606-1619, 2019.

20. Jiang P and Mizushima N: LC3- and p62-based biochemical methods for the analysis of autophagy progression in mammalian cells. Methods 75: 13-18, 2015

21. He F, Ru X and Wen T: NRF2, a transcription factor for stress response and beyond. Int J Mol Sci 21: 4777, 2020.

22. Baird L and Yamamoto M: The molecular mechanisms regulating the KEAP1-NRF2 pathway. Mol Cell Biol 40: e00099-20. 2020 .

23. Unoki T, Akiyama $M$ and Kumagai Y: Nrf2 activation and its coordination with the protective defense systems in response to electrophilic stress. Int J Mol Sci 21: 545, 2020.

24. Menegon S, Columbano A and Giordano S: The dual roles of NRF2 in cancer. Trends Mol Med 22: 578-593, 2016.

25. Zhao Q, Mao A, Yan J, Sun C, Di C, Zhou X, Li H, Guo R and Zhang H: Downregulation of Nrf2 promotes radiation-induced apoptosis through Nrf2 mediated Notch signaling in non-small cell lung cancer cells. Int J Oncol 48: 765-773, 2016.

26. Meng QT, Chen R, Chen C, Su K, Li W, Tang LH, Liu HM, Xue R, Sun Q, Leng Y, et al: Transcription factors Nrf2 and NF-kappaB contribute to inflammation and apoptosis induced by intestinal ischemia-reperfusion in mice. Int J Mol Med 40: 1731-1740, 2017.

27. Wan ZH, Jiang TY, Shi YY, Pan YF, Lin YK, Ma YH, Yang C, Feng XF, Huang LF, Kong XN, et al: RPB5-mediating protein promotes cholangiocarcinoma tumorigenesis and drug resistance by competing with NRF2 for KEAP1 binding. Hepatology 71 2005-2022, 2020.

28. Kitamura $\mathrm{H}$ and Motohashi H: NRF2 addiction in cancer cells. Cancer Sci 109: 900-911, 2018

29. Ji XJ, Chen SH, Zhu L, Pan H, Zhou Y, Li W, You WC, Gao CC, Zhu JH, Jiang K, et al: Knockdown of NF-E2-related factor 2 inhibits the proliferation and growth of U251MG human glioma cells in a mouse xenograft model. Oncol Rep 30: 157-164, 2013.

30. Taguchi K and Yamamoto M: The KEAP1-NRF2 System in Cancer. Front Oncol 7: 85, 2017.

31. Livak KJ and Schmittgen TD: Analysis of relative gene expression data using real-time quantitative PCR and the 2(-Delta Delta C(T)) method. Methods 25: 402-408, 2001

32. Tang Z, Li C, Kang B, Gao G, Li C and Zhang Z: GEPIA: A web server for cancer and normal gene expression profiling and interactive analyses. Nucleic Acids Res 45: W98-W102, 2017.

33. Yang WT, Li GH, Li ZY, Feng S, Liu XQ, Han GK, Zhang H, Qin XY, Zhang R, Nie QM, et al: Effect of corilagin on the proliferation and $\mathrm{NF}-\kappa \mathrm{B}$ in U251 glioblastoma cells and U251 glioblastoma stem-like cells. Evid Based Complement Alternat Med 2016: 1418309, 2016

34. Basso J, Miranda A, Sousa J, Pais A and Vitorino C: Repurposing drugs for glioblastoma: From bench to bedside. Cancer Lett 428 173-183, 2018.

35. Hau DK, Zhu GY, Leung AK, Wong RS, Cheng GY, Lai PB, Tong SW, Lau FY, Chan KW, Wong WY, et al: In vivo anti-tumour activity of corilagin on Hep3B hepatocellular carcinoma. Phytomedicine 18: 11-15, 2010.

36. Pham AT, Malterud KE, Paulsen BS, Diallo D and Wangensteen $\mathrm{H}$ : DPPH radical scavenging and xanthine oxidase inhibitory activity of Terminalia macroptera leaves. Nat Prod Commun 6: 1125-1128, 2011.

37. Gaudreault R and Mousseau N: Mitigating Alzheimer's disease with natural polyphenols: A review. Curr Alzheimer Res 16: 529-543, 2019.

38. Moraes LS, Donza MR, Rodrigues AP, Silva BJ, Brasil DS, Zoghbi M, Andrade EH, Guilhon GM and Silva EO Leishmanicidal activity of (+)-phyllanthidine and the phytochemical profile of Margaritaria nobilis (Phyllanthaceae). Molecules 20: 22157-22169, 2015.

39. Li Y, Xu D, Sun A, Ho SL, Poon CY, Chan HN, Ng OT, Yung KK, Yan H, Li HW, et al: Fluoro-substituted cyanine for reliable in vivo labelling of amyloid- $\beta$ oligomers and neuroprotection against amyloid- $\beta$ induced toxicity. Chem Sci 8: 8279-8284, 2017.

40. Adesina SK, Idowu O, Ogundaini AO, Oladimeji H, Olugbade TA, Onawunmi GO and Pais M: Antimicrobial constituents of the leaves of Acalypha wilkesiana and Aacalypha hispida. Phytother Res 14: 371-374, 2000.

41. Yeo SG, Song JH, Hong EH, Lee BR, Kwon YS, Chang SY, Kim SH, Lee SW, Park JH and Ko HJ: Antiviral effects of Phyllanthus urinaria containing corilagin against human enterovirus 71 and Coxsackievirus A16 in vitro. Arch Pharm Res 38: 193-202, 2015. 
42. Burapadaja S and Bunchoo A: Antimicrobial activity of tannins from Terminalia citrina. Planta Med 61: 365-366, 1995.

43. Teodoro GR, Brighenti FL, Delbem AC, Delbem ÁC, Khouri S, Gontijo AV, Pascoal AC, Salvador MJ and Koga-Ito CY: Antifungal activity of extracts and isolated compounds from Buchenavia tomentosa on Candida albicans and non-albicans. Future Microbiol 10: 917-927, 2015.

44. Li X, Deng Y, Zheng Z, Huang W, Chen L, Tong Q and Ming Y: Corilagin, a promising medicinal herbal agent. Biomed Pharmacother 99: 43-50, 2018.

45. Guo YJ, Luo T, Wu F, Liu H, Li HR, Mei YW, Zhang SL, Tao JY, Dong JH, Fang Y, et al: Corilagin protects against HSV1 encephalitis through inhibiting the TLR2 signaling pathways in vivo and in vitro. Mol Neurobiol 52: 1547-1560, 2015.

46. Komori A, Yatsunami J, Suganuma M, Okabe S, Abe S, Sakai A, Sasaki K and Fujiki H: Tumor necrosis factor acts as a tumor promoter in BALB/3T3 cell transformation. Cancer Res 53: $1982-1985,1993$

47. Okabe S, Suganuma M, Imayoshi Y, Taniguchi S, Yoshida T and Fujiki H: New TNF-alpha releasing inhibitors, geraniin and corilagin, in leaves of Acer nikoense, Megusurino-ki. Biol Pharm Bull 24: 1145-1148, 2001

48. Tong F, Zhang J, Liu L, Gao X, Cai Q, Wei C, Dong J, Hu Y, Wu G and Dong X: Corilagin attenuates radiation-induced brain injury in mice. Mol Neurobiol 53: 6982-6996, 2016.

49. Lu MC, Ji JA, Jiang ZY and You QD: The Keap1-Nrf2-ARE pathway as a potential preventive and therapeutic target: An update. Med Res Rev 36: 924-963, 2016.

50. Chen HH, Chang HH, Chang JY, Tang YC, Cheng YC, Lin LM Cheng SY, Huang CH, Sun MW, Chen CT, et al: Enhanced B-Raf-mediated NRF2 gene transcription and HATs-mediated $\mathrm{NRF} 2$ protein acetylation contributes to ABCC1-mediated chemoresistance and glutathione-mediated survival in acquired topoisomerase II poison-resistant cancer cells. Free Radic Biol Med 113: 505-518, 2017

51. Beidler DR, Chang JY, Zhou BS and Cheng YC: Camptothecin resistance involving steps subsequent to the formation of protein-linked DNA breaks in human camptothecin-resistant KB cell lines. Cancer Res 56: 345-353, 1996.

52. Ferguson PJ, Fisher MH, Stephenson J, Li DH, Zhou BS and Cheng YC: Combined modalities of resistance in etoposide-resistant human KB cell lines. Cancer Res 48: 5956-5964, 1988.

53. Xu B, Wang S, Li R, Chen K, He L, Deng M, Kannappan V, Zha J, Dong $\mathrm{H}$ and Wang W: Disulfiram/copper selectively eradicates AML leukemia stem cells in vitro and in vivo by simultaneous induction of ROS-JNK and inhibition of NF- $\mathrm{KB}$ and Nrf2. Cell Death Dis 8: e2797, 2017.

54. Yang F, Wang Y, Xue J, Ma Q, Zhang J, Chen YF, Shang ZZ, Li QQ, Zhang SL and Zhao L: Effect of Corilagin on the miR-21/smad7/ ERK signaling pathway in a schistosomiasis-induced hepatic fibrosis mouse model. Parasitol Int 65: 308-315, 2016.

55. Zhou X, Xiong J, Lu S, Luo L, Chen ZL, Yang F, Jin F, Wang Y, Ma Q, Luo YY, et al: Inhibitory effect of corilagin on miR-21-regulated hepatic fibrosis signaling pathway. Am J Chin Med 47: 1541-1569, 2019.

56. Rami A and Kögel D: Apoptosis meets autophagy-like cell death in the ischemic penumbra: Two sides of the same coin? Autophagy 4: 422-426, 2008.

57. Siddiqui WA, Ahad A and Ahsan H: The mystery of BCL2 family: Bcl-2 proteins and apoptosis: an update. Arch Toxicol 89: 289-317, 2015
58. Yousefi S, Perozzo R, Schmid I, Ziemiecki A, Schaffner T, Scapozza L, Brunner T and Simon HU: Calpain-mediated cleavage of Atg 5 switches autophagy to apoptosis. Nat Cell Biol 8: 1124-1132, 2006.

59. Booth LA, Tavallai S, Hamed HA, Cruickshanks N and Dent P: The role of cell signalling in the crosstalk between autophagy and apoptosis. Cell Signal 26: 549-555, 2014.

60. Wang $\mathrm{Z}$ and Guo S: Nrf2/HO-1 mediates the neuroprotective effect of mangiferin on early brain injury after subarachnoid hemorrhage by attenuating mitochondria-related apoptosis and neuroinflammation. Sci Rep 7: 11883, 2017.

61. Wu J, Zhang L, Li H, Wu S and Liu Z: Nrf2 induced cisplatin resistance in ovarian cancer by promoting CD99 expression. Biochem Biophys Res Commun 518: 698-705, 2019.

62. Liu P, Rojo de la Vega M, Sammani S, Mascarenhas JB Kerins M, Dodson M, Sun X, Wang T, Ooi A, Garcia JG, et al: RPA1 binding to NRF2 switches ARE-dependent transcriptional activation to ARE-NRE-dependent repression. Proc Natl Acad Sci USA 115: E10352-E10361, 2018.

63. Buendia I, Michalska P, Navarro E, Gameiro I, Egea J and León R: Nrf2-ARE pathway: An emerging target against oxidative stress and neuroinflammation in neurodegenerative diseases. Pharmacol Ther 157: 84-104, 2016.

64. Shaw P and Chattopadhyay A: Nrf2-ARE signaling in cellular protection: Mechanism of action and the regulatory mechanisms. J Cell Physiol 235: 3119-3130, 2020.

65. Jiang T, Harder B, Rojo de la Vega M, Wong PK, Chapman E and Zhang DD: p62 links autophagy and Nrf2 signaling. Free Radic Biol Med 88: 199-204, 2015.

66. Chen RH, Chen YH and Huang TY: Ubiquitin-mediated regulation of autophagy. J Biomed Sci 26: 80, 2019.

67. Narayanan S, Cai CY, Assaraf YG, Guo HQ, Cui Q, Wei L, Huang JJ, Ashby Jr CR and Chen ZS: Targeting the ubiquitin-proteasome pathway to overcome anti-cancer drug resistance. Drug Resist Updat 48: 100663, 2020.

68. Im E, Yoon JB, Lee HW and Chung KC: Human telomerase reverse transcriptase (hTERT) positively regulates $26 \mathrm{~S}$ proteasome activity. J Cell Physiol 232: 2083-2093, 2017.

69. Lokireddy S, Kukushkin NV and Goldberg AL: cAMP-induced phosphorylation of 26S proteasomes on Rpn6/PSMD11 enhances their activity and the degradation of misfolded proteins. Proc Natl Acad Sci USA 112: E7176-E7185, 2015.

70. Roh JL, Kim EH, Jang H and Shin D: Nrf2 inhibition reverses the resistance of cisplatin-resistant head and neck cancer cells to artesunate-induced ferroptosis. Redox Biol 11: 254-262, 2017.

71. Wang Y, Mandal AK, Son YO, Pratheeshkumar P, Wise JT, Wang L, Zhang Z, Shi X and Chen Z: Roles of ROS, Nrf2, and autophagy in cadmium-carcinogenesis and its prevention by sulforaphane. Toxicol Appl Pharmacol 353: 23-30, 2018.

72. Kovac S, Angelova PR, Holmström KM, Zhang Y, Dinkova-Kostova AT and Abramov AY: Nrf2 regulates ROS production by mitochondria and NADPH oxidase. Biochim Biophys Acta 1850: 794-801, 2015.

This work is licensed under a Creative Commons Attribution-NonCommercial-NoDerivatives 4.0 International (CC BY-NC-ND 4.0) License. 